\title{
Évolution des connaissances sur la membrane du globule gras du lait : synthèse bibliographique
}

\author{
Sabine DANTHINE ${ }^{\mathrm{a}}$, Christophe BlECKER ${ }^{\mathrm{a} *}$, Michel PAQUOT ${ }^{\mathrm{a}}$, \\ Nadia INNOCENTE ${ }^{\mathrm{b}}$, Claude DEROANNE ${ }^{\mathrm{a}}$ \\ ${ }^{a}$ Unité de Technologie des Industries Agro-alimentaires, \\ Faculté Universitaire des Sciences Agronomiques de Gembloux, passage des Déportés 2, \\ 5030 Gembloux, Belgique \\ b Dipartimento di Scienze degli Alimenti, Università degli studi di Udine, \\ Via Marangoni 97, 33100 Udine, Italie
}

(Reçu le 24 mars 1999 ; accepté le 23 août 1999)

\begin{abstract}
Progress in milk fat globule membrane research: a review. The milk fat globule is essentially an oil droplet enclosed in a protective 'membrane', partially derived from the plasma membrane of the lactating cell, and which assures the dispersion of the milk fat in the milk plasma. This protective coat is generally called 'the milk fat globule membrane' (MFGM). This review examines the present state of knowledge on the MFGM. The topics dealt with are: origin and formation, isolation from milk, composition (lipids and proteins), structure (a new model is proposed), functional properties and the role that the MFGM plays in protection against lipolysis.
\end{abstract}

milk fat globule membrane / isolation / composition / structure / functional property

Résumé - Les globules gras du lait sont essentiellement des gouttelettes lipidiques enrobées d'une enveloppe protectrice. Cette dernière est partiellement dérivée de la membrane plasmique de la cellule sécrétrice ; de par sa nature, elle assure la dispersion de la matière grasse laitière, hydrophobe, dans le plasma du lait, hydrophile. Cette enveloppe protectrice est généralement appelée la «membrane du globule gras du lait » ou «milk fat globule membrane » (MFGM). Cette synthèse bibliographique fait le point sur les connaissances actuellement acquises à propos de cette membrane du globule gras du lait. Les différents sujets abordés concernent son origine et sa formation, son isolement à partir du lait, sa composition générale (protéines et lipides), sa structure - un nouveau modèle est proposé -, ses propriétés fonctionnelles, ainsi que le rôle qu'elle joue dans la protection du lait contre la lipolyse.

membrane du globule gras du lait / isolement / composition / structure / propriété fonctionnelle

* Correspondance et tirés à part

Tél. : (32) 81622308 ; fax : (32) 81601767 ; e-mail : blecker.c@ fsagx.ac.be 


\section{INTRODUCTION}

Le lait de vache contient de 3 à $5 \%$ de matière grasse dispersée sous forme de globules sphériques dont le diamètre varie de 0,1 à $20 \mu \mathrm{m}$, avec une valeur moyenne de 3 à $5 \mu \mathrm{m}$. Ces globules gras sont hétérogènes ; ils sont essentiellement constitués d'une microgoutte de triglycérides, partiellement cristallisés à température ambiante, entourée d'une fine membrane communément appelée « la membrane du globule gras du lait » ou « milk fat globule membrane » (MFGM). Cette enveloppe protectrice est un assemblage complexe de protéines, de phospholipides, de glycoprotéines, de lipides neutres, d'enzymes et autres composés mineurs. Elle agit comme un émulsifiant naturel permettant à la matière grasse, hydrophobe, de demeurer dispersée dans le plasma du lait, hydrophile et contribue de ce fait au maintien de l'émulsion.

Certains aspects concernant la MFGM n'ont plus fait l'objet de publications ces dernières années ; ils ont été largement repris dans des articles de synthèse assez anciens, faisant encore figure de référence $[3,39,42]$.

Au contraire, d'autres aspects comme la structure, les propriétés fonctionnelles et le rôle de la membrane dans la protection contre la lipolyse font l'objet de recherches plus récentes. Ces points seront dès lors particulièrement développés dans cette synthèse bibliographique.

\section{ORIGINE DE LA MFGM}

\subsection{Origine et formation des globules gras}

Les globules gras du lait se développent dans les cellules de l'épithélium sécrétoire mammaire. En effet, le réticulum endoplasmique est l'endroit où sont synthétisées à la fois les protéines et les triglycérides [7, $36]$; ces triglycérides sont ensuite accumulés sous forme de petites gouttes dans le cytoplasme. Ces gouttes lipidiques de 1 à
$5 \mu \mathrm{m}$ de diamètre, abondantes dans les cellules sécrétrices, sont appelées CLDs (« cytoplasmic lipid droplets »-gouttelettes lipidiques cytoplasmiques) ; ce sont les précurseurs des globules gras du lait. Une description complète de la formation des globules gras du lait se trouve dans l'article de synthèse de Mather et Keenan de 1998 [36].

\subsection{Sécrétion des globules gras du lait - formation de la MFGM}

Dès 1959, Bargmann et Knoop ont observé que lorsque les globules bourgeonnent hors des cellules sécrétrices, ils sont enveloppés d'une membrane [4]. Le mécanisme de sécrétion des globules gras le plus largement accepté actuellement est la migration des gouttelettes lipidiques (CLDs) à travers le cytoplasme de la cellule sécrétrice vers le pôle apical de cette cellule. Lorsque les CLDs atteignent cette surface apicale, elles sont graduellement recouvertes par la membrane plasmique de la cellule, jusqu'à ce qu'il ne reste qu'un fin col non refermé. La sécrétion proprement dite a lieu quand les membranes du col fusionnent entre elles ; la goutte lipidique est alors expulsée dans le lumen alvéolaire.

Ce phénomène serait facilité par une glycoprotéine : la butyrophiline. Ceci est d'autant plus probable que la butyrophiline est acylée et se lie fortement aux phospholipides; de plus, elle a tendance à s'associer à une autre protéine, la xanthine oxydase, pour former un complexe qui pourrait être impliqué dans la liaison de la bicouche membranaire à la goutte lipidique [36]. En outre, durant le bourgeonnement des globules gras, il reste un espace de 10 à $20 \mathrm{~nm}$ entre la surface externe de la goutte lipidique et la face cytoplasmique de la membrane plasmique [36] qui se remplit de matériel «electron-dense ». Cette zone correspond à la zone de couverture présentant un arrangement hexagonal paracristallin en microscopie électronique ; cette couche est constituée de protéines, notamment de la xanthine oxydase et de la butyrophiline. 
La membrane du globule gras peut donc être perçue comme une structure multicouche complexe consistant en une couche interne de protéines et de lipides originaires des CLDs, et une bicouche externe dérivée de la membrane plasmique de la cellule sécrétrice [36].

\section{ISOLEMENT DE LA MEMBRANE}

Le matériel membranaire peut être isolé à partir du lait selon différentes techniques, mais les rendements d'extraction et la composition des isolats obtenus dépendent fortement de la méthode utilisée.

Il est donc primordial de choisir la technique d'isolement répondant le mieux aux objectifs de l'étude réalisée. Ces objectifs sont, par exemple : (i) la sélectivité de la méthode : ceci implique l'obtention de composants membranaires exempts de contaminants (autres constituants du lait, notamment les caséines) ; (ii) le rendement d'extraction : la méthode doit alors permettre l'extraction d'un maximum de matériel membranaire ; (iii) une extraction totale des différents constituants : le matériel doit être extrait sans la moindre perte d'un quelconque élément constitutif de la membrane ; (iv) l'étude de la structure membranaire : la méthode doit être non ou peu dénaturante afin de préserver la structure initiale.

\subsection{Méthodes dites classiques}

Les méthodes d'extraction les plus classiques comportent 5 étapes. Il s'agit de la séparation des globules gras du plasma, de l'élimination des composants du lait écrémé restants (lavage de la crème), de la maturation de la crème obtenue, de la déstabilisation de l'émulsion et de la récupération des fragments de membrane.

\subsubsection{Lavage}

La séparation des globules gras est aisément réalisée par centrifugation de lait non réfrigéré et non pasteurisé afin d'éviter des changements de composition au sein de la membrane.

La crème ainsi obtenue est débarrassée des constituants du lait écrémé par de simples lavages à l'aide de solutions adéquates. Anderson et Brooker [2] ont montré que 3 lavages sont suffisants pour éliminer les composants du lait écrémé et que des lavages supplémentaires entraîneraient trop de pertes en matériel membranaire (phospholipides et protéines).

Basch et al. [6] ont, quant à eux, étudié l'effet de la composition de la solution de lavage sur les isolats obtenus ; l'effet majeur se remarque au niveau de la composition protéique de la membrane, principalement en ce qui concerne les caséines. En effet, seuls les tampons contenant un minimum de $0,15 \mathrm{~mol} \cdot \mathrm{L}^{-1}$ de $\mathrm{NaCl}$ permettent l'élimination des caséines. La présence du $\mathrm{NaCl}$ dans les solutions lavantes permet, en outre, d'éviter une chute de la force ionique du milieu lors de la dilution des crèmes. Cette dernière entraînerait une déstabilisation précoce des globules gras et ainsi une perte de matériel membranaire.

Une variante de ces techniques traditionnelles nécessitant des cycles répétés de centrifugation pour obtenir des globules gras débarrassés de composants provenant du lait écrémé a été mise au point par Patton et Huston en 1986 ; elle permet d'isoler des globules gras « purifiés » en une seule étape [43]. Elle consiste à placer du lait frais non réfrigéré, additionné de saccharose à raison de $5 \mathrm{~g} \cdot 100 \mathrm{~mL}^{-1}$, sous une phase aqueuse tampon ou solution saline ou encore eau dans un tube à centrifugation ; ce tube est fermé et centrifugé à $1500 \mathrm{~g}$ durant $20 \mathrm{~min}$. La couche contenant la matière grasse « purifiée » est collectée en surface de la phase aqueuse. La comparaison de la teneur en protéines, phospholipides et cholestérol des globules gras obtenus à l'aide de cette méthode à celle des globules gras obtenus par la méthode classique faisant appel à des cycles successifs de lavage et centrifugation, montre que les 2 techniques permettent 
d'obtenir des globules gras de composition similaire. Ainsi, cette méthode permet d'obtenir des résultats semblables à ceux obtenus par la méthode traditionnelle de façon beaucoup plus simple et beaucoup plus rapide étant donné le nombre réduit de manipulations. En outre, cette méthode est beaucoup moins dénaturante envers les globules gras.

Pour certaines études spécifiques, les globules gras lavés peuvent être utilisés tels quels. C'est le cas notamment des études d'activité enzymatique, de mobilité électrophorétique, de marquage des constituants externes. Par contre, pour d'autres études, il est nécessaire de déstabiliser l'émulsion soit par voie physique, soit par voie physicochimique de façon à séparer la membrane du cœur lipidique. Cette déstabilisation a généralement lieu après une maturation de la crème. En effet, le refroidissement de la crème lors de la maturation physique permet la cristallisation partielle de la matière grasse ; sans cette cristallisation, le barattage de la crème serait impossible.

\subsubsection{Déstabilisation}

Il existe 2 méthodes de déstabilisation par voie physique : le barattage et la technique du « freeze-thawing » (congélation décongélation).

Le barattage ou agitation vigoureuse de la crème, initialement proposé par Kitchen [32], demeure la méthode la plus couramment utilisée à ce jour $[6,19,20,22,26]$.

La technique du « freeze-thawing », utilisée notamment par Kim et Nawar [30] et Luo et Snow [34], consiste à congeler et décongeler successivement l'échantillon afin de déstabiliser l'émulsion. Il faut noter que ces opérations sont plus efficaces lorsqu'elles sont effectuées lentement [42].

Les méthodes physico-chimiques consistent, quant à elles, à utiliser un détergent ou un solvant afin de déstabiliser les globules. Différents agents peuvent être utilisés : par exemple le Triton-X100 [27, 41] ; des sels biliaires comme le taurodéoxycholate [44] ; le diméthyle sulfoxyde ou le diméthyle formamide [14].

\subsubsection{Récupération}

Après déstabilisation des globules gras, les fragments membranaires se retrouvent dans la phase aqueuse. La méthode la plus communément utilisée pour collecter ces fragments est l'ultracentrifugation. Selon Patton et Keenan [42], une ultracentrifugation à $100000 \mathrm{~g}$ pendant 60 à 90 min permet de récupérer tout le matériel membranaire relargué par barattage ou congélation-décongélation sous forme d'un culot dense.

Toutefois, d'autres alternatives ont aussi été envisagées afin de collecter la membrane par centrifugation à plus faible vitesse. Kanno et al. [25] utilisent du sulfate d'ammonium à $50 \%$ de saturation afin de précipiter la membrane ; le culot est récupéré par centrifugation à $10000 \mathrm{~g}$ pendant $30 \mathrm{~min}$. Cette technique nécessite cependant l'élimination du sulfate d'ammonium après la récupération de la membrane ; cette élimination est effectuée par dialyse. Kanno et Kim [24] effectuent cette étape en $3 \mathrm{j}$ à $4{ }^{\circ} \mathrm{C}$. Une autre alternative, proposée par les mêmes auteurs, consiste à acidifier le milieu à $\mathrm{pH} 4,8$ puis à le centrifuger à $30000 \mathrm{~g}$ pendant $15 \mathrm{~min}$; les résultats obtenus sont comparables, tant au niveau du rendement que de la composition, à ceux obtenus par ultracentrifugation ou précipitation au sulfate d'ammonium.

\subsection{Technique particulière}

Parallèlement à ces méthodes d'extraction dites classiques, comprenant 5 étapes, McPherson et al. [40] ont mis au point une technique particulière ne nécessitant pas de lavage de la crème avant la déstabilisation de l'émulsion. Les caséines et les protéines du lactosérum sont alors ôtées par centrifugation du matériel extrait par barattage (babeurre et eau de lavage du beurre), 
délicatement déposé sur une solution concentrée de saccharose $(52,5 \%$ poids/ volume de saccharose dans un tampon Tris- $\mathrm{HCl}$ à $\mathrm{pH}$ 7,5). Les fragments membranaires sont ensuite collectés à l'interface eau-saccharose, alors que les protéines du lactosérum demeurent dans le surnageant et que les micelles de caséines migrent dans la solution concentrée de saccharose. Le matériel membranaire collecté à l'interface à l'aide d'une seringue est ensuite dialysé et centrifugé. Il faut toutefois noter que le rendement en matériel membranaire est inférieur de $25 \%$ par rapport aux méthodes plus conventionnelles. Il s'agit d'une méthode particulièrement adaptée à l'isolement de matériel membranaire à partir de produits laitiers dont on ignore la stabilité des globules ou présentant une stabilité globulaire réduite.

Afin de contrôler l'efficacité de l'extraction et ainsi vérifier si elle répond effectivement aux objectifs poursuivis, différentes analyses sont généralement effectuées. La mesure du rendement d'extraction, exprimée en grammes de poids sec membranaire pour $100 \mathrm{~g}$ de matière grasse, est couramment réalisée. Différentes analyses concernant la composition du matériel extrait peuvent également être effectuées, notamment la détermination de la teneur en lipides et en protéines. De plus, une électrophorèse permet souvent de vérifier la nature des protéines présentes dans l'extrait obtenu. À l'aide de cette analyse, il est possible de s'assurer, d'une part, que les protéines de la membrane sont présentes et d'autre part, qu'il n'existe aucun contaminant protéique venant du lait écrémé comme par exemple des caséines.

\section{COMPOSITION GÉNÉRALE DE LA MEMBRANE}

Dans la plupart des analyses de composition des isolats de membrane obtenus à l'aide des techniques décrites ci-dessus, la somme des lipides et des protéines dépasse
$90 \%$ du poids sec. Cependant, les proportions relatives de ces 2 composants varient fortement selon les auteurs. Ces variations ont des origines multiples. Elles peuvent notamment provenir du mode de déstabilisation des globules gras, du mode de récupération des fragments membranaires, ainsi que d'autres facteurs non maîtrisables liés à la matière première qu'est le lait. Parmi ces derniers, nous pouvons citer la race et l'âge de l'animal, son régime, son stade de lactation, le facteur saisonnier.

Les plus grandes variations de composition sont observées au sein des lipides neutres et plus particulièrement des triglycérides.

\subsection{Les protéines}

La teneur en protéines varie, selon les auteurs, de 25 à $60 \%$ du poids sec [15, 24, $29,30,41]$.

Les protéines membranaires sont généralement caractérisées par électrophorèse sur gel de polyacrylamide en conditions dénaturantes (SDS-PAGE) ; bien qu'il existe quelques écarts au niveau de l'estimation des poids moléculaires, les profils électrophorétiques obtenus par différents chercheurs présentent une similitude frappante quant à la distribution des polypeptides et glycoprotéines majeurs de la membrane. En effet, en dépit de différences de poids moléculaires calculés et des différences obtenues quant au nombre total de bandes (certaines bandes correspondant aux protéines mineures n'apparaissent pas toujours), 3 classes majeures apparaissent, après révélation au bleu de Coomassie, vers 155 000, 67000 et $44000-48000 \mathrm{~g} \cdot \mathrm{mol}^{-1}$. Le polypeptide correspondant à la classe 155000 , polypeptide 3 selon la nomenclature de Mather et Keenan [35], a été identifié comme étant la xanthine oxydase $[6,17,21]$. Cette enzyme accomplit des réactions d'oxydoréduction variées, notamment l'oxydation de la xanthine en acide urique avec production d'eau oxygénée. Ces petites quantités 
d'eau oxygénée pourraient inhiber le développement de certains micro-organismes [1]. Le polypeptide représentant la classe 67 000, polypeptide 12 selon la nomenclature de Mather et Keenan [35], a, quant à lui, été appelé butyrophiline en raison de son affinité pour les lipides du lait [6, 17]. Cette protéine est insoluble ou très faiblement soluble en solution aqueuse même après un traitement réalisé à l'aide de divers détergents ou dans des solutions de force ionique élevée. La butyrophiline est la glycoprotéine majeure de la MFGM ; elle représente $40 \%$ des protéines totales associées à la membrane, sur base du poids, et $50 \%$ sur base du nombre de molécules [35, 37]. Il s'agit de la seule protéine membranaire dont le gène est exprimé spécifiquement dans les tissus mammaires. Son rôle potentiel dans la sécrétion des globules gras a déjà été souligné mais, à l'heure actuelle, il est encore difficile de définir précisément l'action de cette protéine. En 1981, Franke et al. [17] ont suggéré que cette protéine pourrait jouer le rôle de récepteur facilitant les interactions entre les gouttelettes lipidiques cytoplasmiques et la membrane plasmique apicale. Il faut encore noter que la xanthine oxydase pourrait former un complexe avec la butyrophiline [37]. Ce complexe serait chargé d'assurer la sécrétion correcte des gouttes lipidiques ; ceci confirmerait l'hypothèse initiale de Franke. La suppression de l'expression du gène correspondant à la butyrophiline, par exemple chez la souris, permettrait certainement de progresser dans la détermination du rôle exact de cette protéine.

La troisième classe importante se situe vers 44 000-48 000 [6]. Cette classe, correspondant au polypeptide 16 selon la nomenclature de Mather et Keenan [35], contient une glycoprotéine purifiée et identifiée par Basch et al. [5] : il s'agit de la glycoprotéine B. Cette protéine étant exprimée dans le cas de tumeurs du sein, on utilise, en médecine humaine, des anticorps monoclonaux correspondants, afin de détecter ce type de cancers.
Il faut aussi noter qu'il existe, en plus de ces protéines majoritaires, un certain nombre de protéines minoritaires qui possèdent peutêtre des fonctions biologiques importantes, notamment des enzymes. Une description exhaustive des enzymes membranaires se trouve dans l'article de synthèse de McPherson et Kitchen [39].

\subsection{Les lipides}

Les lipides neutres comprennent les triglycérides, les diglycérides, les monoglycérides, les esters et le cholestérol, par opposition aux lipides polaires représentés par les glycolipides et les phospholipides. En fait, la membrane du globule gras, dans son état natif normal (c'est-à-dire dans du lait non réfrigéré) contient des triglycérides, mais probablement en faible quantité [51]. Les grandes quantités de triglycérides généralement retrouvées parmi les constituants membranaires proviennent en grande partie de cristaux de matière grasse du cœur du globule contaminant la membrane durant le processus de barattage. Ces triglycérides co-entrainés avec le matériel membranaire se distinguent des triglycérides totaux du lait, car ils contiennent une plus grande proportion d'acides gras à longue chaîne [49] ; ils sont d'ailleurs qualifiés de triglycérides à haut point de fusion [20]. La proportion de triglycérides associés à la membrane dépend fortement des conditions d'isolement; c'est pourquoi de grandes variations existent quant à la composition lipidique de la membrane.

Les lipides polaires ou complexes comprennent les glycolipides et les phospholipides. Ces derniers ont à la fois des propriétés hydrophiles et hydrophobes, et de ce fait contribuent largement au rôle émulsifiant de la membrane. Les types majeurs de phospholipides présents dans la membrane et leurs pourcentages approximatifs sont : la phosphatidylcholine $35 \%$, la phosphatidyléthanolamine $30 \%$, la sphingomyéline $25 \%$, le phosphatidylinositol $5 \%$, la phosphatidylsérine $3 \%$ [15]. 
La composition moyenne de la matière grasse membranaire est reprise dans le tableau I ; la composition des lipides neutres exprimée en pourcentage des lipides totaux est reprise dans le tableau II.

\section{LA STRUCTURE \\ DE LA MEMBRANE DU GLOBULE GRAS DU LAIT}

De par sa fonction d'émulsifiant naturel, il est logique de concevoir une structure de la membrane du globule gras du lait qui soit asymétrique, puisqu'elle constitue le lien physique entre deux phases totalement différentes. D'une part, elle est adjacente au cœur lipidique du globule gras et d'autre part, elle fait face à la phase aqueuse du lait. De ce fait, il est normal de trouver les composants les plus hydrophobes vers l'intérieur, du côté lipidique, et les constituants les plus hydrophiles vers l'extérieur, en contact avec le plasma.

La combinaison des différentes techniques existant actuellement ainsi que des données concernant l'origine et la sécrétion des globules gras (voir § 2) ont permis d'obtenir de nombreux éléments concernant cette structure.
Grâce à la microscopie électronique, on a pu observer l'existence d'une couche « electron-dense » sur la face interne de la membrane. Des études immuno-microscopiques menées par Franke et al. [17] et biochimiques menées par Freudenstein [18] ont permis de montrer que la xanthine oxydase et la butyrophiline sont 2 constituants majeurs de cette zone.

La microscopie électronique associée à la technique du cryodécapage ou « freezeetching » a permis à Buchheim [7] d'observer la surface externe du globule gras. Dans le lait fraîchement sécrété, l'apparence de cette surface varie fortement. En effet, certains globules sont couverts par une surface relativement lisse. Mais on peut aussi, assez fréquemment, observer des domaines façonnés de façon originale en surface des globules : ils sont en forme de plaques avec des protubérances circulaires ou allongées en périphérie. De plus, ces domaines varient largement en taille et en aspect ; ils peuvent ainsi couvrir de grandes proportions de la surface globulaire. Ces domaines particuliers résultent de la présence de constituants situés entre la bicouche membranaire et le cœur lipidique. Afin d'accroître les connaissances concernant l'organisation structurelle de ces domaines, Buchheim [7] a

Tableau I. Composition moyenne de la matière grasse membranaire.

Table I. Mean composition of membranous milk fat.

\begin{tabular}{lcl}
\hline Classe lipidique & $\begin{array}{c}\text { Pourcentage rapporté } \\
\text { aux lipides totaux }\end{array}$ & Référence \\
\hline Lipides polaires & $42,6 \pm 6,8$ & Patton [41] \\
(phospholipides et glycolipides) & $15-54$ & McPherson et Kitchen [39] \\
& $24 \pm 2$ & Dapper et al. [14] \\
& 34,3 & Houlihan et al. [19] \\
Acides gras libres & $35,5 \pm 1,5$ & Danthine (résultat non publié, 1998) \\
& $0,6-6,3$ & Patton et Keenan [42], Keenan et al. [29] \\
Lipides neutres & $4-6$ & Danthine (résultat non publié ; 1998) \\
& $56-80$ & Keenan et al. [29], Kim et Nawar [30] \\
& $57-59$ & Danthine (résultat non publié, 1998) \\
\hline
\end{tabular}


Tableau II. Composition des lipides neutres exprimée en pourcentage des lipides totaux. Table II. Neutral lipids composition (\% of total lipids).

\begin{tabular}{lcl}
\hline Classe lipidique & $\begin{array}{c}\text { Pourcentage rapporté } \\
\text { aux lipides totaux }\end{array}$ & Référence \\
\hline Triglycérides & $51-68$ & Anderson et Cawston [3] \\
& $37,3 \pm 9$ & Patton [41] \\
& $48,4 \pm 2,6$ & Chen et Nawar [9] \\
& $45,8 \pm 1$ & Danthine (résultat non publié, 1998) \\
Diglycérides & 9 & Keenan et Patton [28] \\
& $8,5 \pm 0,5$ & Danthine (résultat non publié, 1998) \\
Monoglycérides & traces & Keenan et Patton [28] \\
& $0,7 \pm 0,2$ & Danthine (résultat non publié, 1998) \\
Esters & $0,1-0,8$ & Patton et Keenan [42], Keenan et al. [29] \\
Cholestérol & $0,2-5,2$ & Patton et Keenan [42] \\
& $6,1 \pm 0,4$ & Patton [41] \\
& $0,2-2,0$ & Keenan et Patton [28] \\
& $1,7 \pm 0,2$ & Danthine (résultat non publié, 1998) \\
\hline
\end{tabular}

étudié le résultat du clivage à l'interface entre l'enveloppe et le cœur du globule gras. Ce clivage, qui se produit fréquemment lorsque les échantillons de lait sont congelés lors du traitement préparatoire à l'analyse par cryodécapage, permet de voir d'une part, que le façonnage de ces domaines laisse une empreinte dans la matière grasse (face externe des triglycérides) et d'autre part, que ces domaines protéiques sont hautement organisés suivant un ordre paracristallin avec une symétrie principalement hexagonale (face interne du matériel membranaire). Ce type d'arrangement de protéines en structure hexagonale n'est d'ailleurs pas rare dans les systèmes biologiques; on le trouve, en effet, souvent associé aux membranes cellulaires [38].

La MFGM posséderait donc une structure multicouche complexe, avec une couche constituée de lipides et de protéines en contact avec le cœur lipidique et une bicouche typique d'une membrane biologique. Des recherches récentes confirment cette hypothèse. En effet, Deeth [15] rapporte que des études réalisées à l'aide de phospholipases spécifiques ont permis de mettre en évidence une disposition asymétrique des phospholipides membranaires, comme dans le cas d'autres membranes biologiques, et notamment celle des globules rouges. Ainsi, on trouve principalement situés du côté externe la phosphatidylcholine, la sphingomyéline ; et principalement sur la face interne, la phosphatidyléthanolamine, le phosphatidylinositol, la phosphatidylsérine.

Tous ces éléments nous amènent, pour conclure ces aspects structurels, à discuter du modèle proposé pour représenter schématiquement la MFGM. Jusqu'à présent, 3 modèles principaux ont été suggérés : celui de King [31], celui de Morton-Hayashi cité par Peerebom [45], et celui de McPherson et Kitchen [39]. Nous proposons de nuancer ce dernier modèle en plaçant l'orientation des différents phospholipides constitutifs de la membrane selon les données apportées par Deeth [15], les glycoprotéines ainsi que le complexe xanthine oxydase-butyrophiline (Fig. 1). 


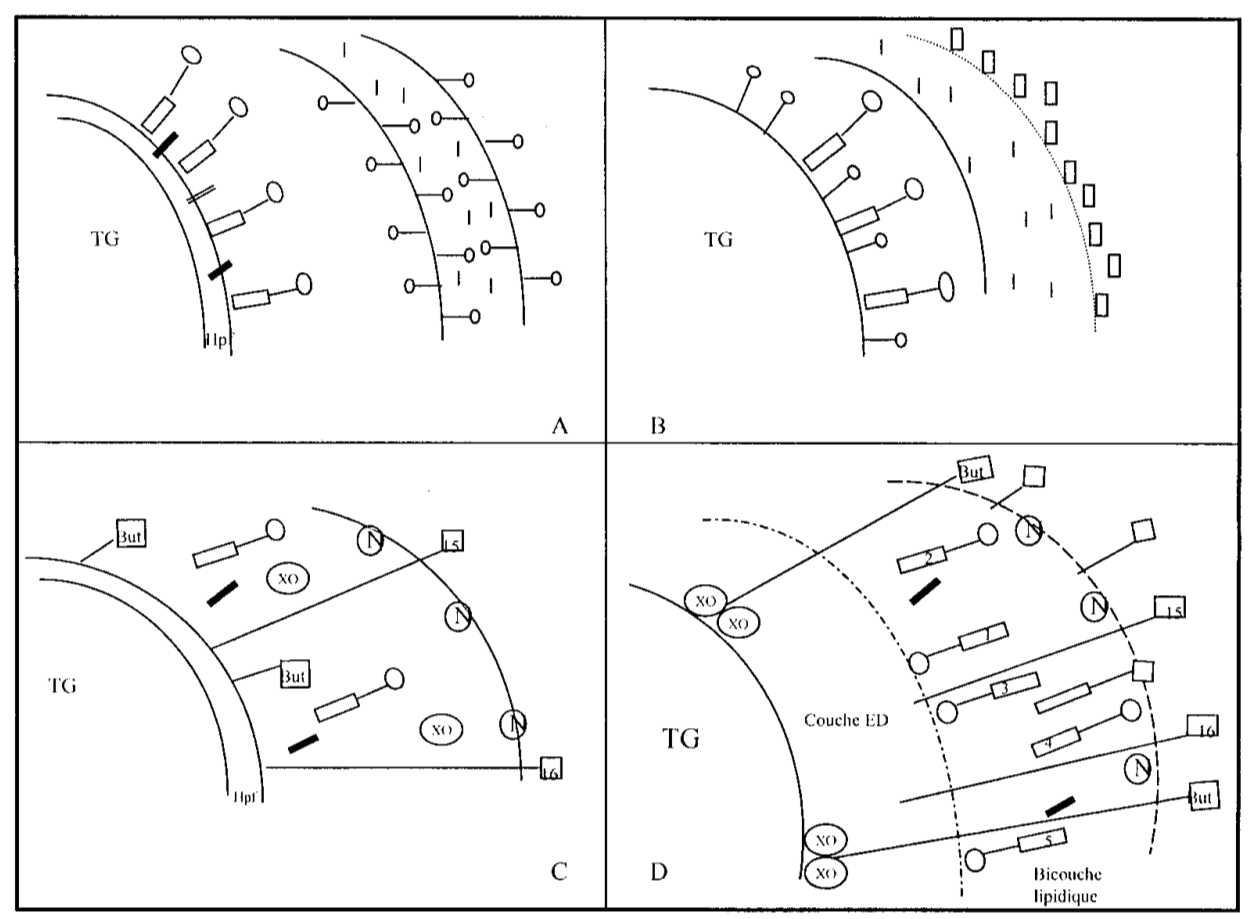

A : inspiré de King [31] ; B : inspiré de Morton - Hayashi cité par Peereboom [45];

$\mathrm{C}$ : inspiré de McPherson et Kitchen [39] ; D : modèle actualisé (1999).

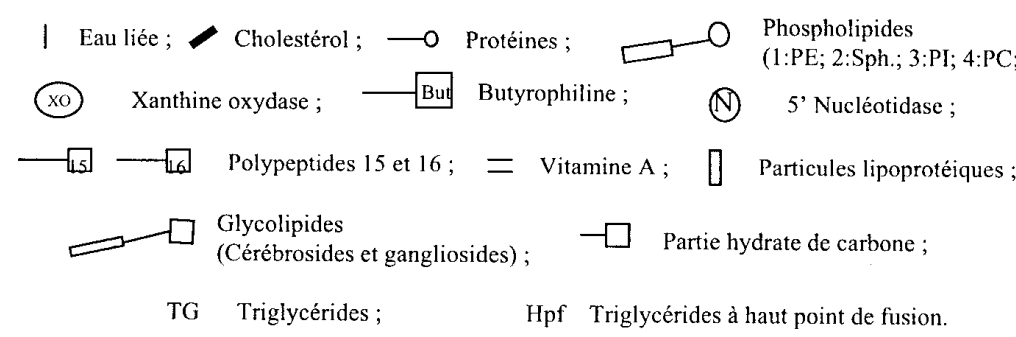

Figure 1. Évolution du modèle proposé pour schématiser la membrane du globule gras du lait.

Figure 1. Various stages in the evolution of the proposed model for milk fat globule membrane.

\section{PROPRIÉTÉS \\ FONCTIONNELLES DE \\ LA MEMBRANE DU GLOBULE GRAS DU LAIT}

\subsection{Propriétés de surface de la MFGM}

Les propriétés de surface de la MFGM ont été étudiées en 1982 par Phipps et Temple [46] « in situ », à l'aide d'une méthode utilisant une micropipette de verre pour produire des distorsions contrôlées de globules liquides. Il s'agit d'une technique qui a aussi été employée par Rand et Burton [47] pour étudier les propriétés d'érythrocytes. La tension interfaciale des globules gras obtenue à l'aide de cette méthode est inférieure à $2 \mathrm{mN} \cdot \mathrm{m}^{-1}$. Cette valeur extrêmement basse - la tension interfaciale matière grasse laitière liquide/plasma du lait 
est, quant à elle, de $15 \mathrm{mN} \cdot \mathrm{m}^{-1}$ [52] - justifie pleinement le pouvoir émulsifiant de la MFGM.

Chazelas et al. [8] ont, quant à eux, comparé à l'aide d'une balance à film de Langmuir les propriétés de surface de matériel membranaire à celles de caséinate de sodium. Il ressort de cette étude d'une part, que lorsque ces 2 substances sont en compétition, une augmentation de la pression d'homogénéisation améliore l'adsorption des caséines sur la surface des globules gras et d'autre part, que la stabilité des laits et des crèmes pourrait être améliorée en ajoutant des isolats de membrane, provenant par exemple du fractionnement du babeurre, au lieu d'augmenter la pression d'homogénéisation.

Certaines équipes de chercheurs se sont récemment intéressées aux propriétés mécaniques de monocouches formées à partir de l'ensemble de la MFGM ou à partir de certains constituants isolés. Ainsi, Kristensen et al. [33] ont étudié les propriétés mécaniques de monocouches de sphingomyéline provenant de la MFGM, ainsi que les interactions de cette fraction avec la xanthine oxydase. En 1997, Innocente et al. [20] ont étudié les propriétés mécaniques de monocouches formées à partir de l'étalement d'une fraction soluble de membrane extraite à partir de crèmes non pasteurisées. Cette étude réalisée à l'aide d'une balance à film de Langmuir a permis de déterminer l'influence de la température (de 4 à $40^{\circ} \mathrm{C}$ ) sur les propriétés mécaniques du film ainsi formé. Ainsi, il apparaît que la fraction de membrane étudiée a des propriétés mécaniques différentes lorsqu'elle est étalée à l'interface air/eau à basse température, c'està-dire inférieure à $15^{\circ} \mathrm{C}$, qu'à plus haute température (jusqu'à $40^{\circ} \mathrm{C}$ ). Ceci peut être partiellement expliqué par le haut pourcentage de triglycérides à haut point de fusion présents dans cette fraction de membrane. L'étude par analyse calorimétrique différentielle de cette fraction soluble de mem- brane montre qu'il existe des différences significatives de pourcentage de triglycérides cristallisés pour de faibles variations de température, dans l'intervalle $4-40{ }^{\circ} \mathrm{C}$. Ainsi, 86,5\% de triglycérides sont cristallisés à $4{ }^{\circ} \mathrm{C}$ et seulement $28,6 \%$ à $40{ }^{\circ} \mathrm{C}$. De plus, Dufour et al. ont montré que la température de transition des phospholipides membranaires se situe entre 10 et $30^{\circ} \mathrm{C}$ [16]. Ces études démontrent clairement l'influence de la température sur les propriétés de surface du matériel membranaire.

\subsection{Propriétés émulsifiantes}

Kanno [23] a étudié les propriétés émulsifiantes de la membrane en reconstituant des globules gras. Les variations de concentration de la membrane (de 20 à $80 \mathrm{mg} \cdot \mathrm{g}^{-1}$ de matière grasse) affectent significativement les propriétés des émulsions ainsi reconstituées (activité émulsifiante, foisonnement, stabilité moussante, stabilité émulsifiante). Ce même auteur, en 1991 [26], a poursuivi ces recherches en étudiant les propriétés physico-chimiques d'émulsions reconstituées à l'aide de cette même membrane. Les propriétés étudiées sont la viscosité, l'adsorption de protéines sur les globules gras et la distribution de taille des globules reconstitués. L'observation de la distribution de taille des globules, à l'aide d'un microscope optique et d'un analyseur de taille de particules à diffraction laser, montre que le diamètre moyen des globules diminue plus ou moins linéairement avec l'augmentation de concentration de membrane. La conclusion de l'ensemble de ces études indique que la MFGM est un émulsifiant naturel intéressant, et qu'il est possible de reconstituer des globules ayant une stabilité similaire à celle du lait natif si l'on emploie une concentration de membrane de l'ordre de $80 \mathrm{mg} \cdot \mathrm{g}^{-1}$ de matière grasse (MG). Il est à noter que la concentration de MFGM optimale est nettement supérieure à la concentration naturellement présente dans les produits laitiers (1,5 g.100 $\left.\mathrm{g}^{-1} \mathrm{MG}\right)$; 
cette différence peut trouver son origine d'une part, dans l'absence d'autres constituants pouvant amener de la matière sèche non grasse (comme des caséines, du lactose, des protéines sériques) dans la formulation étudiée. D'autre part, il faut noter que la membrane ainsi reconstituée diffère de la membrane naturelle puisqu'à l'origine, elle dérive d'un processus biologique complexe faisant intervenir plusieurs étapes (voir § 2). En effet, la membrane des globules gras natifs est un assemblage asymétrique complexe dérivant de la membrane plasmique de la cellule sécrétrice. En outre, il faut encore noter que la stabilité à la coalescence des globules gras natifs est principalement liée à la présence de chaînes glucidiques des glycoprotéines et des glycolipides membranaires sur la face externe de la membrane. En effet, la présence de telles chaînes assure une stabilité à la coalescence suite à un effet stérique.

L'utilisation de membrane pure comme ingrédient fonctionnel n'est toutefois pas envisageable du point de vue économique. Par contre, l'utilisation de babeurre industriel riche en éléments membranaires, et donc influencé par les propriétés la membrane, comme ingrédient fonctionnel dans le secteur alimentaire suscite de plus en plus d'intérêt. Cet ingrédient naturel pourrait amener des propriétés spécifiques dans une large gamme de produits alimentaires formulés ; une telle utilisation est une voie intéressante de valorisation de ce sous-produit inhérent à la fabrication industrielle du beurre.

Les études les plus récentes ont de ce fait un caractère appliqué, étant donné qu'elles portent directement sur les propriétés fonctionnelles des babeurres.

Ainsi, Corredig et Dalgleish [11] ont évalué les propriétés émulsifiantes de membranes extraites à partir de babeurres industriels ainsi que celles des babeurres pris dans leur totalité. Les isolats de membrane obtenus à partir de babeurres industriels présentent des propriétés émulsifiantes nettement inférieures à celles du babeurre « entier ». Le traitement thermique ainsi que le barattage industriel affectent significativement le comportement de la membrane, notamment suite à la fixation d'une quantité importante de $\beta$-lactoglobuline sur cette membrane durant la pasteurisation des crèmes [19]. La quantité de $\beta$-lactoglobuline associée à la membrane augmente d'ailleurs avec l'augmentation de température, principalement si elle dépasse $65^{\circ} \mathrm{C}$ [12]. Bien que le rôle de la membrane dans la stabilité des crèmes soit clairement établi, il s'avère que le traitement thermique subi lors du processus industriel de fabrication du beurre altère considérablement ses propriétés émulsifiantes. Afin de mettre en évidence l'effet produit par le traitement thermique, Corredig et Dalgleish [13] ont étudié les propriétés d'émulsions préparées avec du babeurre ou de la MFGM provenant de crèmes ayant subi ou non un traitement thermique avant barattage. Lorsque les émulsions sont préparées avec du babeurre, la distribution des diamètres des globules ne diffère pas quel que soit le traitement subi (ou sans traitement) ; au contraire, lorsque les émulsions sont préparées avec de la MFGM, on constate que les propriétés émulsifiantes sont beaucoup moins bonnes lorsque la crème a subi un traitement HTST, en comparaison avec une crème non traitée thermiquement ou chauffée jusqu'à 60-62 ${ }^{\circ} \mathrm{C}$. Ceci montre que les caséines présentes dans le babeurre sont les protéines principalement adsorbées à l'interface.

De plus, la quantité de fer présente dans la MFGM diminue avec l'augmentation de la température de chauffage. Cette perte de fer pourrait être en relation avec la xanthine oxydase. En effet, il s'agit d'une métalloflavoprotéine et cette perte de fer pourrait être en relation avec une perte de métal de cette protéine. Ce relargage de fer pourrait rendre les cystéines des protéines membranaires aptes à des échanges de disulfures avec d'autres protéines contenant des cystéines ( $\beta$-lactoglobuline, $\kappa$-caséine) [13]. 
Le chauffage de la crème avant la fabrication du beurre constitue donc une étape critique concernant les propriétés fonctionnelles de la MFGM. Cette réflexion peut d'ailleurs être mise en relation avec les expériences de Innocente et al. [20] montrant que les propriétés interfaciales du film membranaire varient en fonction de la température.

\section{RÔLE POTENTIEL \\ DE LA MFGM \\ DANS LA PROTECTION CONTRE LA LIPOLYSE}

Dans le lait de vache, la lipolyse n'a normalement pas lieu - ou très peu - en dépit de la présence de lipoprotéine lipase naturelle. Elle se développe cependant rapidement si ce même lait est agité ou homogénéisé. On considère généralement que la membrane constitue une barrière physique naturelle réduisant l'accès des lipases aux triglycérides du lait [10].

Shimizu et al. [48] ont utilisé une émulsion reconstituée à base de matière grasse laitière stabilisée à l'aide de gomme arabique. L'effet des lipases, qu'elles soient microbiennes ou pancréatiques, est remarquablement inhibé s'ils ajoutent de la MFGM à l'émulsion ; cet effet est cependant perdu si la MFGM est préalablement traitée à l'aide de trypsine. Ceci démontre le rôle important des protéines membranaires dans l'inhibition de l'hydrolyse enzymatique des triglycérides des globules gras du lait. De plus, Sundheim et BengtsonnOlivecrona [50] ont montré que l'extraction des protéines membranaires à l'aide de guHCl (guanidine hydrochlorure) ou $\mathrm{MgCl}_{2}$ rend le substrat beaucoup plus accessible à la lipoprotéine lipase et que l'addition de ces protéines restaure le rôle protecteur de la membrane et stoppe la lipolyse. De même, l'ajout de cette fraction protéique à du lait entier diminue l'action des lipases.

Cependant, suite à la complexité de la composition de la membrane, le mécanisme inhibiteur demeure incompris bien qu'il soit admis que les protéines membranaires y jouent un rôle non négligeable.

\section{CONCLUSION}

La physico-chimie particulière du lait fascine les chercheurs depuis de nombreuses années, ce qui explique le nombre important d'études réalisées concernant la membrane du globule gras du lait. Cette membrane apparaît actuellement comme un système très complexe dont l'extraction nécessite de nombreuses précautions. Il n'existe d'ailleurs pas un mode d'extraction bien défini ; au contraire, différentes techniques peuvent être utilisées en fonction des objectifs recherchés. De plus, la composition de la membrane est assez variable en fonction de la technique d'extraction. Il est donc souhaitable de combiner différentes techniques afin de la caractériser. De même, il peut être très utile d'utiliser les techniques d'investigation des membranes biologiques pour progresser dans la connaissance de la MFGM (RMN, FTIR, FAM, ...).

Le lait représente un remarquable système naturel qu'il serait intéressant d'imiter. Il s'agit en effet d'une émulsion naturelle très stable dont la connaissance des propriétés structurales et tensioactives pourrait faire progresser notamment dans la conception de produits alimentaires émulsionnés stables. De plus, la caractérisation des propriétés fonctionnelles de la membrane et des babeurres ouvre la voie vers de nouvelles perspectives de valorisation industrielle de ce sous-produit en tant qu'ingrédient fonctionnel naturel.

\section{REMERCIEMENTS}

Cette étude a été réalisée avec l'aide du ministère de la Région wallonne, en application du programme de formation et d'impulsion à la recherche scientifique et technologique. 


\section{RÉFÉRENCES}

[1] Alais C., Science du lait. Principes des techniques laitières, Éd. Sepaic, Paris, 1984.

[2] Anderson M., Brooker B.E., Loss of material during the isolation of milk fat globule membrane, J. Dairy Sci. 58 (1975) 1442-1448.

[3] Anderson M., Cawston T.W., Reviews of the progress of the dairy science: the milk-fat globule membrane, J. Dairy Res. 42 (1975) 459-483.

[4] Bargmann W., Knoop A., Über die Morphologie der Milchsekretion. Licht und electronenmikroskopische Studien an der Milchdrüse der Ratte, Z. Zellforsch. 49 (1959) 344-388.

[5] Basch J.J., Farrell H.M. Jr., Greenberg R., Identification of the milk fat globule membrane proteins. I. Isolation and partial characterization of glycoprotein B, Biochim. Biophys. Acta 448 (1976) 589-598.

[6] Basch J.J., Greenberg R., Farrell H.M. Jr., Identification of the milk fat globule membrane proteins. II. Isolation of major proteins from electrophoretic gels and comparison of their amino acid compositions, Biochim. Biophys. Acta 830 (1985) 127-135.

[7] Buchheim W., Membranes of milk fat globules ultrastructural, biochemical and technological aspects, Kiel. Milchwirtsch. Forschungsber. 38 (1986) 227-246.

[8] Chazelas S., Razafindralambo H., Dumont de Chassart Q., Paquot M., Surface properties of the milk fat globule membrane : competition between casein and membrane material, in : Dickinson E., Lorient D. (Eds.), Food macromolecules and colloids, Roy. Soc. Chemistry, Cambridge, Royaume Uni, 1995, pp. 95-98.

[9] Chen Z.Y., Nawar W.W., Role of milk fat globule membrane in autoxidation of milk fat, J. Food Sci. 56 (1991) 398-401.

[10] Chilliard Y., Lamberet G., La lipolyse dans le lait : les différents types, mécanismes, facteurs de variation, signification pratique, Lait 64 (1984) 544-578.

[11] Corredig M., Dalgleish D.G., Isolates from industrial buttermilk: emulsifying properties of material derived from the milk fat globule membrane, J. Agric. Food Chem. 45 (1997) 4595-4600.

[12] Corredig M., Dalgleish D.G., Buttermilk properties in emulsions with soybean oil as affected by fat globule membrane-derived proteins, J. Food Sci. 63 (1998) 476-480.

[13] Corredig M., Dalgleish D.G., Effect of heating of cream on the properties of milk-fat globule membrane isolates, J. Agric. Food Chem. 46 (1998) 2533-2540.

[14] Dapper C.H., Valivullah H.M., Keenan T.W., Use of polar aprotic solvents to release membranes from milk lipid globules, J. Dairy Sci. 70 (1987) 760-765.
[15] Deeth H., The role of phospholipids in the stability of milk fat globules, Australian Food Industry Science Center, Milkfat update conf., 1997.

[16] Dufour E., Subirade M., Loupil F., Riaublanc A., Whey proteins modify the phase transition of milk fat globule phospholipids, Lait 79 (1999) 217-228.

[17] Franke W.W., Heid H.W., Grund C., Winter S. Freudenstein C., Schurid E., Jarasch E.D., Keenan T.W., Antibodies to the major insoluble milk fat globule membrane associated proteins: specific location in apical regions of lactating epithelial cells, J. Cell. Biol. 89 (1981) 485-494.

[18] Freudenstein C., Keenan T.W., Eigel W.N., Sasaki M., Stadler J., Franke W.W., Preparation and characterization of the inner coat material associated with fat globule membranes from bovine and human milk, Exp. Cell Res. 118 (1979) 277-294.

[19] Houlihan A.V., Goddard P.A., Nottingham S.M., Kitchen B.J., Masters C.J., Interactions between the BMFGM and skim milk components on heating whole milk, J. Dairy Res. 59 (1992) 187-195.

[20] Innocente N., Blecker C., Deroanne C., Paquot M., Langmuir film balance study of the surface properties of a soluble fraction of milk fat globule membrane, J. Agric. Food Chem. 45 (1997) 1559-1563.

[21] Jarasch E.D., Grund C., Brudder G., Heid H.W., Keenan T.W., Franke W.W., Localization of xanthine oxidase in mammary-gland epithelium and capillary endothelium, Cell 25 (1981) 67-92.

[22] Jensen S.K., Nielsen K.N., Tocopherols, retinol, $\beta$-carotene and fatty acids in fat globule core and cows' milk, J. Dairy Res. 63 (1996) 565-574.

[23] Kanno C., Emulsifying properties of bovine milk fat globule membrane in milk fat emulsion: conditions for the reconstitution of fat globules, J. Food Sci. 54 (1989) 1534-1539.

[24] Kanno C., Kim D.H., A simple procedure for the preparation of bovine milk fat globule membrane and a comparison of its composition, enzymatic activities, and electrophoretic properties with those preparated by other methods, Agric. Biol. Chem. 54 (1990) 2845-2854.

[25] Kanno C., Shimizu M., Yamauchi K., Isolation and physicochemical properties of a soluble glycoprotein fraction of milk fat globule membrane, Agric. Biol. Chem. 39 (1975) 1835-1842.

[26] Kanno C., Schimomura Y., Takano E., Physicochemical properties of milk fat emulsions stabilized with bovine milk fat globule membrane, J. Food Sci. 56 (1991) 1219-1223.

[27] Keenan T.W., Heid A.W., Tight attachment of fatty acids to proteins associated with milk lipid globule membrane, Eur. J. Cell Biol. 26 (1982) 270-276. 
[28] Keenan T.W., Patton S., The stucture of milk: implications for sampling and storage, A. The milk lipid globule membrane, in: Jensen R.G. (Ed.), Handbook of milk composition, Academic Press Inc., San Diego, CA, USA, 1995, pp. 5-50.

[29] Keenan.T.W., Moon T.W., Dylewsky D.P., Lipid globules retain membrane material after homogenization, J. Dairy Sci. 66 (1983 196-203.

[30] Kim S.K., Nawar W.W., Oxidative interactions of cholesterol in the milk fat globule membrane, Lipids 27 (1992) 928-932.

[31] King N.M., The milk fat globule membrane and some associated phenomena, Commonwealth Agricultural Bureaus, Farnham Royal, Bucks, England, 1955

[32] Kitchen B.J., A comparison of the properties of membranes isolated from bovine skim milk and cream, Biochim. Biophys. Acta 356 (1974) 257-269.

[33] Kristensen D., Nylander T., Rasmussen J.T., Paulsson M., Carlsson A., Bovine milk sphingomyelin at the air/water interface and its interaction with xanthine oxydase, Proc. int. meeting on milk proteins: structure and functions, Wadahl, Norvège, 6-8 mars 1996.

[34] Luo X.Y., Snow L.D., Detergent interference with Lowry assay of bovine milk fat globule membrane protein, Biochem. Arch. 13 (1997) 319-326.

[35] Mather I.H., Keenan T.W., Studies on the struc ture of milk fat globule membrane, J. Membrane Biol. 21 (1975) 65-85.

[36] Mather I.H., Keenan T.W., Origin and secretion of milk lipids, J. Mam. Gland Biol. Neoplasia 3 (1998) 259-273.

[37] Mather I.H., Jack L.J.W., A review of the molecular and cellular biology of butyrophilin, the major protein in bovine milk fat globule membrane, J. Dairy Sci. 76 (1993) 3832-3850.

[38] Maurel M.-A., La membrane plasmique, in: Biologie de la cellule, Breal Eds., Rosny, France, 1994, pp. 125-167.

[39] McPherson A.V., Kitchen B.J., Reviews of the progress of dairy science: the bovine milk fat globule membrane - its formation, composition, structure and behaviour in milk and dairy products, J. Dairy Res. 50 (1983) 107-133.
[40] McPherson A.V., Dash M.C., Kitchen B.J., Isolation of bovine milk fat globule membrane material without prior removal of caseins and whey proteins, J. Dairy Res. 51 (1984) 113-121.

[41] Patton S., Release of remnant plasma membrane from milk fat globules by Triton X-100, Biochim. Biophys. Acta 688 (1982) 727-734.

[42] Patton S., Keenan T.W., The milk fat globule membrane, Biochim. Biophys. Acta 415 (1975) 273-309.

[43] Patton S., Huston G.E., A method for isolation of milk fat globules, Lipids 21 (1986) 170-174

[44] Patton S., Borgstrom B., Stemberger B.H., Welsch U., Release of membrane from milk fat globules by conjugated bile salts, J. Pediatr. Gastroenterol. Nutr. 5 (1986) 262-267.

[45] Peereboom C.J., Fette, Seifen Austrich 71 (1969) 314.

[46] Phipps L.W., Temple D.M., Surface properties of milk fat globules : interfacial tension studies, J. Dairy Res. 49 (1982) 61-72.

[47] Rand R.P., Burton A.C., Mechanical properties of the red cell membrane. I. Membrane stiffness and intracellular pressure, Biophys. J. 4 (1964) 115-135.

[48] Shimizu M., Miyaji H., Yamaguchi K., Inhibition of lipolysis by milk fat globule membrane materials in model milk fat emulsion, Agric. Biol. Chem. 46 (1982) 795-799.

[49] Sharma K.C., Kumari A., Sareen V.K., Singh S. Lipid composition of fat globule membrane from buttermilk and butter serum of buffalo and cow milk, Milchwissenschaft 42 (1987) 439-441.

[50] Sundheim G., Bengtsson-Olivecrona G., Hydrolysis of milk fat by lipoprotein lipase: inhibition by proteins extracted from milk fat globule membrane, J. Dairy Sci. 70 (1987) 1815-1821.

[51] Walstra P., High-melting triglycerides in the milk fat globule membrane; an artefact? Neth. Milk Dairy J. 28 (1974) 3-9.

[52] Walstra P., Jenness R., Colloidal and surface phenomena, in: Dairy Chemistry and Physics, J. Wiley \& Sons, New York, USA, 1984 pp. 211-228. 